\title{
Pleuroparenchymal fibroelastosis as a complication of chemotherapy: A case report
}

\author{
ANA CLÁUDIA RODRIGUES VIEIRA ${ }^{1}$, JOANA RAQUEL MONTEIRO FERRA ${ }^{1}$, \\ JOANA SOFIA VICENTE DE CARVALHO ${ }^{1}$, NATÁLIA MELO ${ }^{2}$, PATRÍCIA CAETANO MOTA ${ }^{2,3}$, \\ HÉLDER NOVAIS E BASTOS ${ }^{2-4}$, JOSÉ MIGUEL PEREIRA ${ }^{5}$, \\ CONCEIÇÃO SOUTO MOURA ${ }^{6}$ and ANTÓNIO MORAIS ${ }^{2-4}$ \\ ${ }^{1}$ Pulmonology Department, Egas Moniz Hospital, 1349-019 Lisbon; ${ }^{2}$ Pulmonology Department, \\ Hospital Center of São João; ${ }^{3}$ Faculty of Medicine, University of Oporto, 4200-319 Porto; \\ ${ }^{4}$ Institute for Molecular and Cell Biology/Institute for Research and Innovation in Health, 4200-135 Porto; \\ ${ }^{5}$ Imaging Department and ${ }^{6}$ Pathology Department, Hospital Center of São João, 4200-319 Porto, Portugal
}

Received September 15, 2019; Accepted January 29, 2021

DOI: $10.3892 / \mathrm{mco} .2022 .2504$

\begin{abstract}
Pleuroparenchymal fibroelastosis (PPFE) is a rare and recently described distinct pattern of lung apical fibrosis involving the upper lobe parenchyma and pleural dome. PPFE has definable and reproducible clinical, radiological and histopathological criteria, which allowed its classification as an independent interstitial lung disease. Several factors have been associated with PPFE, such as chemotherapy, especially with alkylating agents. The authors present a case of a 34-year-old female with previous history of Hodgkin lymphoma treated with first line chemotherapy (doxorubicin, bleomycin, vinblastine and dacarbazine). The patient had no other known comorbidities or relevant exposure to lung irritants. A total of 2 years after completing cancer treatment, the patient developed clinical and radiological features of PPFE. Given their previous history of malignancy, a biopsy of the lesion was obtained, which confirmed the diagnosis of PPFE. The authors present this case to raise awareness of this disease and to demonstrate that PPFE can develop months to years following chemotherapy treatment. Moreover, to date, none of these chemotherapy agents have been associated with the development of PPFE.
\end{abstract}

\section{Introduction}

Pleuroparenchymal fibroelastosis (PPFE) is a rare form of interstitial lung disease (ILD), characterized by upper zone

Correspondence to: $\operatorname{Dr}$ Ana Cláudia Rodrigues Vieira, Pulmonology Department, Egas Moniz Hospital, 126 Junqueria Road, 1349-019 Lisbon, Portugal

E-mail: anavieira33@hotmail.com

Key words: pleuroparenchymal fibroelastosis, chemotherapy, doxorubicin, bleomycin, vinblastine, dacarbazine pleural fibrosis with subpleural intra-alveolar fibrosis and alveolar septal elastosis. It was first described in 2004 by Frankel et $a l$ and is considered to be an independent entity among ILDs in the 2013 Classification of Idiopathic Interstitial Pneumonias by the American Thoracic Society/European Respiratory Society $(1,2)$.

PPFE affects both sexes and may be diagnosed in young patients. The clinical course varies as it can progress rapidly in certain cases or have an indolent course (3).

Although its physiopathology remains unclear, certain factors have been associated with PPFE, including chemotherapy. Several cases previously described the association between PPFE and drugs used to treat malignancy, especially alkylating drugs, such as cyclophosphamide and carmustine (3-4). Given the predicted increasing prevalence of patients treated with chemotherapeutic agents, PPFE may become more common in this population (5). However, there is no effective pharmacological treatment and lung transplant must be considered in certain cases. Hence, it is important to raise awareness of this disease among clinicians.

\section{Case report}

A female patient (age, 34 years) was diagnosed with nodular sclerosing Hodgkin lymphoma in 2016 (stage II-A). The patient was treated in Hospital Center of São João (Porto, Portugal) with first line chemotherapy (doxorubicin, bleomycin, vinblastine and dacarbazine) and mediastinal and right axillary radiotherapy. The patient had no other relevant comorbidities (such as auto-immune disease), no history of smoking, no known relevant occupational or environmental exposure and was not prescribed regular medication. In 2018, the patient presented with insidious onset exertional dyspnea and reported a respiratory tract infection that was treated with antibiotics (amoxicillin and clavulanic acid). Physical examination of the patient did not result in any relevant findings. Laboratory blood tests and blood gas analysis were normal. The patient's lung volumes in lung functional tests were within normal range, but 

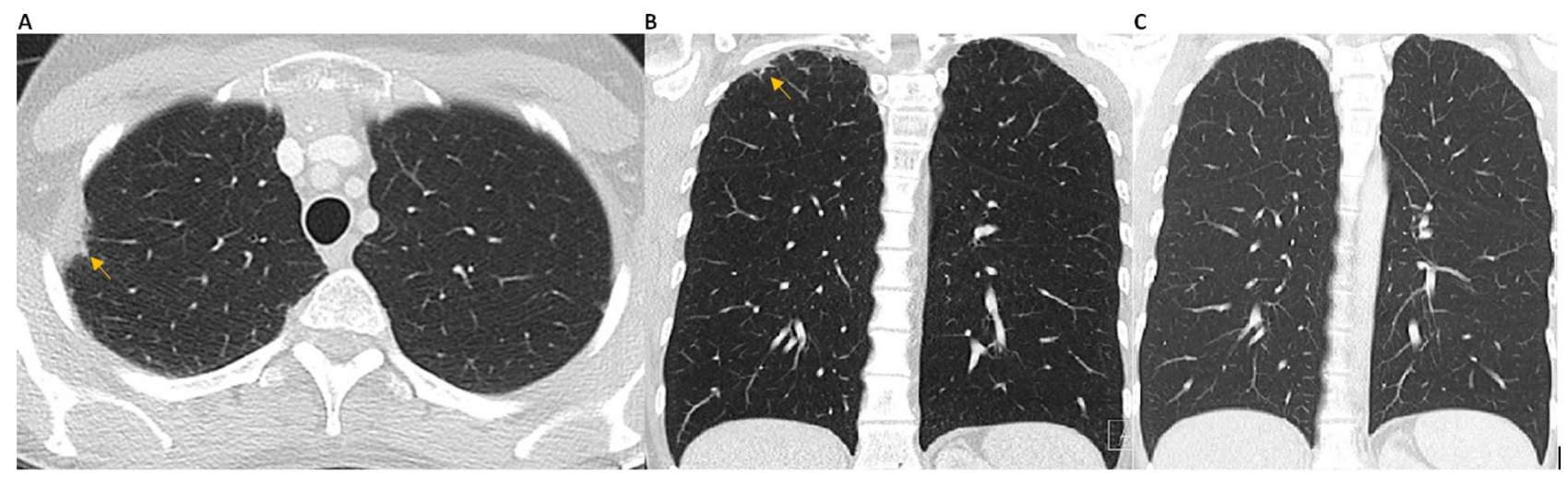

Figure 1. CT images. (A) Axial and (B) coronal chest CT images showing subpleural apical opacities (arrows) with minimal upper-lobe volume loss. Findings are more expressive in the right upper lobe. (C) These changes were not present in previous scans performed 4 years before.

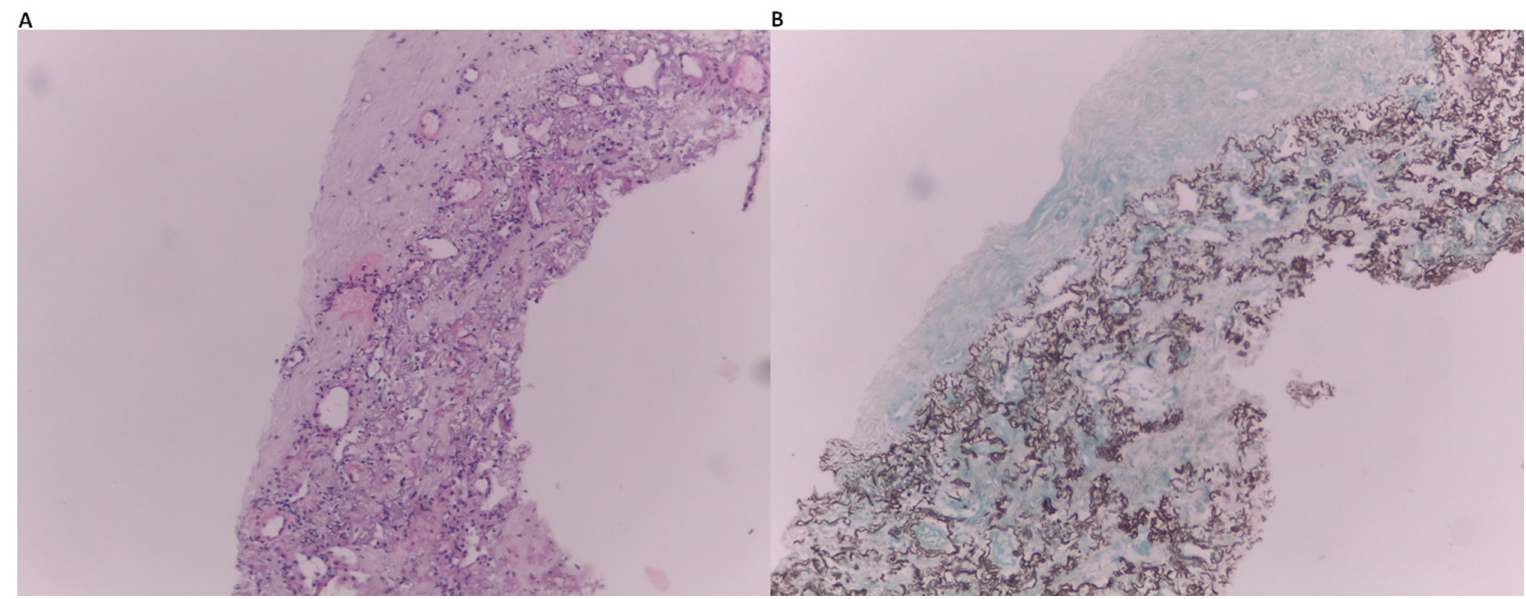

Figure 2. Histology specimens. (A and B) Section of upper lobe biopsy displaying fibrous thickening of the visceral pleura and homogeneous, dense intra-alveolar fibrosis with septal elastosis. Hematoxylin and eosin and stain for elastic fibers were used. Magnification, x100.

the diffusing capacity for carbon monoxide (53\%) and adjusted alveolar volume (54\%) were decreased (6). High-resolution computed tomography (HRCT) of the chest revealed biapical subpleural opacity with interstitial thickening and slight upper lobe volume loss (Fig. 1). These features were not present in previous scans. These changes were subtle, which is also the reason why lung volumes in the respiratory function test were normal. It was hypothesized that the diffusing capacity for carbon monoxide was depressed due to sequelae from previous radiotherapy that resulted in changes in lung function that may not be seen in CT scan. Given her past history of malignancy, a percutaneous transthoracic lung biopsy was performed, which revealed pleural fibrosis and subpleural and parenchymal fibroelastosis (Fig. 2). Following evaluation and discussion among the ILD multidisciplinary team, the patient was diagnosed with PPFE. To date, the patient remains clinically and functionally stable in follow-up appointments. Hence, the patient has not yet been treated with immunosuppressive therapy.

\section{Discussion}

PPFE is a rare and recently described clinical entity. However, the number of diagnosed cases has increased as clinicians become more aware of this disease (7). Although PPFE physiopathology remains unclear, its distinct pattern of fibrosis with definable and reproducible clinical, radiological and histopathological criteria allowed its classification as an independent entity in the 2013 American Thoracic Society and European Respiratory Society classification of Idiopathic Interstitial Pneumonias, specifically as a rare interstitial pneumonia $(8,9)$.

Several factors have been associated with PPFE, including chemotherapy, lung and bone marrow transplant, connective tissue disease and recurrent pulmonary infections (9). It was hypothesized that PPFE may represent a common final pattern of response of the lung to multiple types of injury, such as recurrent infection, allergen exposure and chemotherapy (9). An idiopathic form of PPFE has also been described, as well as familial cases.

Clinically, PPFE equally affects both sexes, with a proportion of patients being relatively young. PPFE develops and progresses rapidly in some cases, whereas other patients present with radiological changes years before symptoms have occurred (9) The most common features of PPFE are dyspnea on exertion, insidious onset dry cough and weight loss (3). Pneumothorax is common due to pleural alterations and can be a complication that is difficult to manage 
following lung biopsy. If radiological features are typical of PPFE and no other diagnosis is suspected, one should consider the risks and benefits of performing a lung biopsy for each individual patient since histology is not mandatory for PPFE diagnosis (10).

Imaging and histological criteria for the diagnosis of PPFE were described in $2012(10,11)$. HRCT imaging criteria of PPFE can be defined as either 'definite' or 'consistent with'. 'Definite' criteria consist of pleural thickening and subpleural fibrosis concentrated in the upper lobes, whereby lower lobe involvement is less marked or absent. 'Consistent with' criteria include upper lobe thickening and subpleural fibrosis; however, the distribution of changes are not concentrated in the upper lobes, or features of coexisting disease are present elsewhere (10).

The histological criteria of PPFE are also defined as either 'definite' and 'consistent with'. 'Definite' criteria include upper zone pleural fibrosis with subjacent intra-alveolar fibrosis, accompanied by alveolar septal elastosis. 'Consistent with' criteria include intra-alveolar fibrosis, but not those associated with significant pleural fibrosis, not predominantly present beneath the pleura or not present in an upper lobe biopsy (10).

Drugs associated with lung injury and pleural effusion, pleuritis, pleural thickening or fibrosis have been reported in this context (7). PPFE has been identified as a complication of chemotherapy with alkylating agents such as cyclophosphamide or carmustine. The disease can develop months to years after treatment and in some cases, it may be difficult to determine a causal connection. Data that support this association are: Pre-therapy normal chest imaging, the existence of reports from different countries over multiple years that describe the same disease associated with the same drugs, different alkylating agents resulting in the same disease and the development of PPFE in young individuals, as fibrotic disease is more common in the elderly.

Bleomycin is used in models of pleural fibrosis and is instilled in the pleural space (7). To the best of our knowledge, bleomycin and the other chemotherapy drugs used in the present case report has not previously been associated with PPFE (7). However, to date, no effective treatment has been able to modify the natural course of PPFE. Corticosteroids and immunosuppressive agents have been used, but with limited efficacy. Lung transplants must be considered in selected patients with advanced disease (8).

Although bleomycin and the other chemotherapy agents used to treat the present patient have not been implied in the etiology of PPFE thus far, this association seems plausible since it was observed in a young patient with no known contact with other possible causative agents and normal chest imaging prior to chemotherapy.

PPFE was diagnosed 2 years after treatment cessation in the present case report. The disease may develop several years after chemotherapy treatment. A study has shown that the interval between the time of drug exposure to onset of PPFE symptoms or radiological features ranges from 6 months to 16 years (7). The present patient also underwent radiotherapy; this treatment can induce pneumonitis/fibrose or organizing pneumonia. However, since PPFE lesions were observed outside of the radiation plan (which excludes the possibility of radiation pneumonitis) and PPFE has never been reported in this scenario, this does not indicate any relevant role of radiation in this particular clinical case.

PPFE has been increasingly diagnosed over recent years due to growing awareness of this disease among clinicians. This will facilitate greater understanding of the etiology of this disease and the development of novel treatment approaches to improve patient quality of life and survival rate in the future.

\section{Acknowledgements}

Not applicable.

\section{Funding}

No funding was received.

\section{Availability of data and materials}

Not applicable.

\section{Authors' contributions}

ACRV, JRMF and JSVDC contributed to the planning, conduct, reporting, conception, design and acquisition of data and drafting the manuscript. AM, NM, PCM, HNEB, JMP and CS contributed to planning, conduct, reporting, conception, design and acquisition of data and revision of the article. All authors read and approved the final manuscript.

\section{Ethics approval and consent to participate}

The authors certify that this study involving a human subject was performed in accordance with the Declaration of Helsinki 1975, as revised in 2000, and was approved by the Ethical Committee of Hospital Center of São João and Faculty of Medicine, University of Oporto (approval no. CE-OP-70-2020). Additionally, informed written consent was obtained from the patient.

\section{Patient consent for publication}

Informed written consent was obtained from the patient.

\section{Competing interests}

The authors declare that they have no competing interests.

\section{References}

1. Frankel SK, Cool CD, Lynch DA and Brown KK: Idiopathic pleuroparenchymal fibroelastosis. Description of a novel clinicopathologic entity. Chest 126: 2007-2013, 2004.

2. Travis W, Costabel U, Hansell DM, King TE Jr, Lynch DA, Nicholson AG, Ryerson CJ, Ryu JH, Selman M, Wells AU, et al: An Official American Thoracic Society/European Respiratory Society Statement: Update of the International Multidisciplinary Classification of the Idiopathic Interstitial Pneumonias. Am J Respir Crit Care Med 88: 733-748, 2013.

3. Portillo K, Guasch Arriaga I and Ruiz-Manzano J: Pleuroparenchymal Fibroelastosis: Is it Also an Idiopathic Entity? Arch Bronconeumol 51: 509-514, 2015 (In English, Spanish). 
4. Beynat-Mouterde C, Beltramo G, Lezmi G, Pernet D, Camus C, Fanton A,Foucher P, Cottin V and Bonniaud P: Pleuroparenchymal fibroelastosis as a late complication of chemotherapy agents. Eur Respir J 44: 523-527,2014.

5. Wilson BE, Jacob S, Yap ML, Ferlay J, Bray F and Barton MB: Estimates of global chemotherapy demands and corresponding physician workforce requirements for 2018 and 2040: A population-based study. Lancet Oncol 20: 769-780, 2019.

6. Pellegrino R, Viegi G, Brusasco V, Crapo RO, Burgos F, Casaburi R, Coates A, van der Grinten CP, Gustafsson P, Hankinson $\mathrm{J}$, et al: Interpretative strategies for lung function tests. Eur Respir J 26: 948-968, 2005.
7. Camus P, Thusen J, Hansell D and Colby T: Pleuroparenchymal fibroelastosis: One more walk on the wild side of drugs? Eur Respir J 44: 289-296, 2014.

8. Bonifazi M, Montero MA and Renzoni EA: Idiopathic Pleuroparenchymal Fibroelastosis. Curr Pulmonol Rep 6: 9-15, 2017

9. Thüsen JH: Pleuroparenchymal Fibroelastosis: Its pathological characteristics. Curr Respir Med Rev 9: 238-247, 2013.

10. Reddy TL, Tominaga M,Hansell DM, von der Thusen J, Rassl D, Parfrey H, Guy S, Twentyman O, Rice A, Maher TM, et al: Pleuroparenchymal Fibroelastosis: A spectrum of histopathological and imaging phenotypes. Eur Respir J 40: 377-385, 2012 . 